\title{
Annihilation signatures of neutron dark decay models in neutron oscillation and proton decay searches
}

\author{
Wai-Yee Keung, ${ }^{a, b}$ Danny Marfatia ${ }^{c}$ and Po-Yan Tseng ${ }^{b, d}$ \\ ${ }^{a}$ Department of Physics, University of Illinois at Chicago, \\ Chicago, Illinois 60607 U.S.A. \\ ${ }^{b}$ Physics Division, National Center for Theoretical Sciences, \\ Hsinchu, Taiwan \\ ${ }^{c}$ Department of Physics and Astronomy, University of Hawaii at Manoa, \\ Honolulu, HI 96822, U.S.A. \\ ${ }^{d}$ Kavli IPMU (WPI), UTIAS, The University of Tokyo, \\ Kashiwa, Chiba 277-8583, Japan \\ E-mail: keung@uic.edu, dmarf8@hawaii.edu, tpoyan1209@gmail.com
}

ABSTRACT: We point out that two models that reconcile the neutron lifetime anomaly via dark decays of the neutron, also predict dark matter-neutron $(\bar{\chi}-n)$ annihilation that may be observable in neutron-antineutron oscillation and proton decay searches at Super-Kamiokande, Hyper-Kamiokande and DUNE. We study signatures of $\bar{\chi} n \rightarrow \gamma \pi^{0}$ (or multi- $\pi^{0}$ ) and $\bar{\chi} n \rightarrow \phi \gamma \pi^{0}$ (or $\phi+$ multi- $\pi^{0}$ ), where $\phi$ is an almost massless boson in one of the two models.

KEYwords: Beyond Standard Model

ArXiv EPrint: 1905.03401 


\section{Contents}

1 Introduction 1

$\begin{array}{llr}2 & \text { Effective interactions and form factor } & 2\end{array}$

3 Model I 3

4 Model II 4

5 Dark matter-nucleon annihilation cross section $\quad 6$

6 Signal events at Super-K, Hyper-K and DUNE 9

7 Results and summary 12

A Cross section calculations $\quad 12$

\section{Introduction}

In the standard model (SM), the neutron almost exclusively decays through beta decay, $n \rightarrow p+e^{-}+\bar{\nu}_{e}$, with $\operatorname{Br}(n \rightarrow p+$ anything $)=1$. The neutron lifetime is measured in bottle experiments and beam experiments which use different methodologies. In bottle experiments, the total neutron lifetime $\tau_{n}^{\text {bottle }}$ is measured by counting the number of neutrons trapped in a container as function of time. On the other hand, beam experiments count the number of protons resulting from neutron decay in a neutron beam. In this case, the neutron lifetime is given by

$$
\frac{1}{\tau_{n}^{\text {beam }}}=-\frac{\operatorname{Br}(n \rightarrow p+\text { anything })}{N_{n}} \frac{d N_{n}}{d t},
$$

where $N_{n}$ is the number of neutrons in the beam. In the SM, the two methods should give the same neutron lifetime. However, there is tension between the bottle [1-3] and beam $[4,5]$ measurements of the neutron lifetime at about the $4 \sigma$ level [6]:

$$
\begin{aligned}
\tau_{n}^{\text {bottle }} & =879.6 \pm 0.6 \mathrm{~s} \\
\tau_{n}^{\text {beam }} & =888.0 \pm 2.0 \mathrm{~s}
\end{aligned}
$$

If neutrons decay through channels without protons in the final state i.e., $\operatorname{Br}(n \rightarrow$ $p+$ anything $)<1$, beam experiments will measure a longer lifetime than bottle experiments since

$$
\tau_{n}^{\text {beam }}=\frac{\tau_{n}^{\text {bottle }}}{\operatorname{Br}(n \rightarrow p+\text { anything })} .
$$


To explain the discrepancy, the neutron decay width into channels without protons must be

$$
\Delta \Gamma(n \rightarrow \text { no proton }) \simeq 7.1 \times 10^{-30} \mathrm{GeV},
$$

which is about $1 \%$ of the total neutron decay width.

Two models, dubbed Model I and Model II, invoking dark decays of the neutron were proposed in refs. $[7,8]$. The basic idea is to introduce a tiny mixing between the neutron and a new particle in the dark sector, which allows the neutron to decay into dark sector particles $\chi$ (a Dirac fermion) and $\phi$ (a scalar) through $n \rightarrow \chi+\gamma$ and $n \rightarrow \chi+\phi$. The $\chi$ couples very weakly with the SM sector so as to not trigger a detectable signal in the beam experiments. Meanwhile, the mass of $\chi$ is restricted in a narrow window,

$$
\text { 937.992 } \mathrm{MeV}<m_{\chi}<938.783 \mathrm{MeV},
$$

to simultaneously satisfy the requirement of ${ }^{9} \mathrm{Be}$ stability and prevent the decay, $\chi \rightarrow$ $p+e^{-}+\bar{\nu}_{e}[7-9]$. These criteria make $\chi$ a good dark matter (DM) candidate if other decay modes in the dark sector are forbidden. Based on these assumptions, $\bar{\chi}-n$ annihilation provides detectable signals with energy of $\mathcal{O}(\mathrm{GeV})$ in Super-Kamiokande (Super-K) or in the future experiments, Hyper-Kamiokande (Hyper-K) and DUNE. The signals are similar to that for proton decay and neutron-antineutron oscillations. For instance in Model I, prompt photon and multi- $\pi^{0}$ signals are obtained from $\bar{\chi} n$ annihilation. Dark matternucleon annihilation has been studied in a broader context in ref. [10].

Note that these models have trouble with neutron star stability [11-13]. The conversion of neutrons to dark matter in the neutron star softens the nuclear equation of state to the point that neutron stars above two solar masses are not possible, which is in contradiction with observations. In a recent paper [14] it was shown that extending Model II with strong repulsive DM-baryon interactions solves the problem. This extension does not contribute additional diagrams to the annihilation signatures we discuss. We view the models of refs. $[7,8,14]$ as examples that produce neutron dark decays that may be extended with complex dark sectors to address various experimental and astrophysical constraints.

The paper is organized as follows. In section 2, we present the relevant effective interactions and parameterize the required form factor. We introduce Model I and -II for the dark decays of the neutron in section 3 and 4, respectively. Signatures of DM-neutron annihilation are discussed in section 5 , and the signal event numbers at underground experiments are estimated in section 6 . We summarize our results in section 7 . We describe some of our cross section calculations in an appendix.

\section{Effective interactions and form factor}

Since the neutron has no electric charge, it couples to the photon via the magnetic dipole interaction,

$$
\mathcal{L}^{\mathrm{eff}} \supset \frac{g_{n} e}{2 m_{n}} F_{\bar{n} \gamma n}\left(Q^{2}\right) \bar{n} \sigma^{\mu \nu} F_{\mu \nu} n,
$$

where $g_{n} \simeq-3.826$ is the neutron $g$-factor and $F_{\bar{n} \gamma n}\left(Q^{2}\right)$ is the corresponding form factor. On the other hand, the pion-neutron and pion-proton effective interactions satisfy the 
isospin symmetry and are given by

$$
\begin{aligned}
\mathcal{L}^{\mathrm{eff}} & \supset \frac{g_{n \pi}}{\sqrt{4 \pi}} F_{\bar{n} \pi n}\left(Q^{2}\right) \bar{N}(\vec{\tau} \cdot \vec{\pi}) i \gamma_{5} N \\
& =\frac{g_{n \pi}}{\sqrt{4 \pi}} F_{\bar{n} \pi n}\left(Q^{2}\right)\left(-\bar{n} i \gamma_{5} n \pi^{0}+\bar{p} i \gamma_{5} p \pi^{0}+\sqrt{2} \bar{p} i \gamma_{5} n \pi^{+}+\sqrt{2} \bar{n} i \gamma_{5} p \pi^{-}\right),
\end{aligned}
$$

where $N=(p, n)^{T}, \vec{\tau} \cdot \vec{\pi}=\sqrt{2}\left(\tau_{-} \pi_{-}+\tau_{+} \pi_{+}\right)+\tau_{3} \pi_{0}, g_{n \pi}=\sqrt{13.54}$ [15], and $F_{\bar{n} \pi n}\left(Q^{2}\right)$ is the form factor. For the $\bar{n} \pi n$ vertex with only $\bar{n}$ off-shell at momentum squared $-Q^{2}$, we parameterize the form factor as

$$
F_{\bar{n} \pi n}\left(Q^{2}\right)=\left(\frac{1-m_{n}^{2} / \Lambda_{n}^{2}}{1+Q^{2} / \Lambda_{n}^{2}}\right)^{y},
$$

where $y$ is an unknown exponent and will be treated as a free parameter determined by data. $y=0$ represents the case of no form factor suppression, while $y=2$ gives a good fit to the electromagnetic form factor of the proton. We determine the value of $y$ by comparing with the experimentally measured $\bar{n} p$ annihilation cross section in the nonrelativistic limit. Here, $\Lambda_{n}$ characterizes the size of the nucleon, and is typically $4 \pi f_{\pi} \approx 1.2 \mathrm{GeV}$. The form factor is normalized to unity for $-Q^{2}=m_{n}^{2}$. Furthermore, we assume the same behavior for the magnetic form factor of the neutron. The complete amplitude also involves the transitional vertices between $\bar{\chi}-n$. We suppose that the form factors $F_{\bar{\chi} \gamma n}\left(Q^{2}\right), F_{\bar{\chi} \pi n}\left(Q^{2}\right)$ and $F_{\bar{n} \pi n}\left(Q^{2}\right)$, all have the same parameterization but may have different values for the exponent $y$, and $y=2$ for the electromagnetic form factor.

\section{Model I}

Model I allows the dark decay $n \rightarrow \chi+\gamma$ by introducing two dark sector particles, a Dirac fermion $\chi$ (whose antiparticle $\bar{\chi}$ we identify as the DM candidate) and a heavy scalar mediator $\Phi$ (color triplet, weak singlet, hypercharge $\frac{Y}{2}=-\frac{1}{3}$ ). The new interaction Lagrangian terms that contribute to $\chi-n$ mixing are [7]

$$
\mathcal{L}_{1} \supset \lambda_{1} \Phi^{*} \chi d_{R}+\lambda_{1}^{\prime} \Phi u_{R} d_{R}+\text { h.c. }
$$

As stated earlier,

$$
\text { 937.992 } \mathrm{MeV}<m_{\chi}<938.783 \mathrm{MeV} .
$$

The colored $\Phi$ must be much heavier than $1 \mathrm{TeV}$ to be compatible with LHC data. It is therefore reasonable to work in the effective theory framework to describe processes at the $\mathrm{GeV}$ scale. The DM-triquark operator is derived by integrating out the heavy scalar $\Phi$ :

$$
\mathcal{L} \subset \frac{\lambda_{1} \lambda_{1}^{\prime}}{m_{\Phi}^{2}}\left(\chi u_{R} d_{R} d_{R}\right)=\frac{\lambda_{1} \lambda_{1}^{\prime}}{m_{\Phi}^{2}} \beta(\chi n)
$$

where form factor $\beta=\left\langle 0\left|u_{R} d_{R} d_{R}\right| n\right\rangle \simeq 0.0144 \mathrm{GeV}^{3}$ [16]. This operator is effectively an off-diagonal mass term between the DM and neutron, leading to a $m_{\Phi}^{2}$ suppressed mixing angle,

$$
\theta \simeq \frac{\varepsilon}{m_{n}-m_{\chi}}
$$

where $\varepsilon \equiv \beta \lambda_{1} \lambda_{1}^{\prime} / m_{\Phi}^{2}$. 


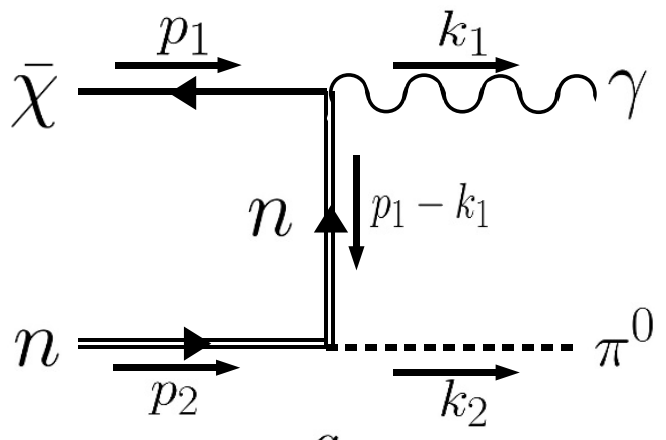

$a$.

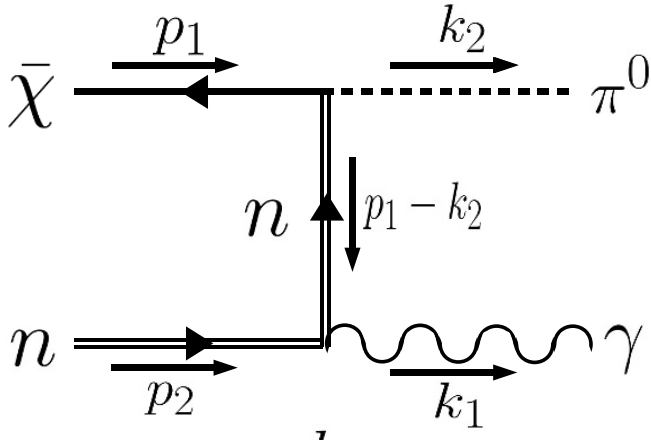

$b$.

Figure 1. Feynman diagrams for $\bar{\chi}+n \rightarrow \gamma+\pi^{0}$ in Model I.

The DM-neutron mixing gives rise to a DM-neutron-photon coupling, which is responsible not only for neutron dark decay $n \rightarrow \chi \gamma$, but also predicts the DM-neutron annihilation channel,

$$
\bar{\chi}+n \rightarrow \gamma+\pi^{0},
$$

as shown in figure 1. A nonrelativistic DM particle in the halo interacts with a static neutron target and produces a photon and pion back-to-back, each with an energy of about a GeV. The topology of the signal is similar to that of the proton decay channel $p \rightarrow e^{+} \pi^{0}$ at experiments like Super-K. Both produce 3 electron-like Cherenkov rings in Super-K [17], and the reconstructed total momentum $P_{\text {tot }}$ tends to be small. However, the reconstructed invariant mass $M_{\text {tot }} \simeq 2 \mathrm{GeV}$ from $\bar{\chi}+n \rightarrow \gamma+\pi^{0}$, is higher than $M_{\text {tot }} \simeq 1 \mathrm{GeV}$ from $p \rightarrow e^{+} \pi^{0}$.

A multi-pion final state can also result from DM-neutron annihilation. It is analogous to $\bar{n}$-nucleon annihilation in the SM which predominantly yields a multi-pion final state. The DM-nucleon annihilation cross section is related to the $\bar{n}$-nucleon cross section via [10]

$$
\sigma\left(\bar{\chi} N \rightarrow \operatorname{multi}-\pi^{0}\right)=\theta^{2} \sigma\left(\bar{n} N \rightarrow \operatorname{multi}-\pi^{0}\right) .
$$

This multi- $\pi^{0}$ signal can be detected in Super-K as well, and the signal kinematics is similar to that for $n-\bar{n}$ oscillations [18]. However, because of the compositeness of the hadron and a lack of experimental measurements of the $\bar{n} \pi n$ form factor, a perturbative calculation using eqs. (2.1), (2.2) cannot give a precise estimate of the annihilation cross section.

To overcome this, we match our calculation to the experimentally measured $\bar{n} p$ annihilation cross section [19-22] and multiply it by the mixing angle square $\theta^{2}$ to obtain the $\bar{\chi}-n$ annihilation cross section. We elaborate on our procedure in section 5 .

\section{Model II}

Model II has a richer structure than Model I with two additional dark sector particles: a Dirac fermion $\tilde{\chi}$ and a complex scalar $\phi$ [7]. After the heavy scalar $\Phi$ is integrated out, $\tilde{\chi}$ mixes with the neutron through the mixing angle

$$
\theta \simeq \frac{\varepsilon}{m_{n}-m_{\tilde{\chi}}}
$$




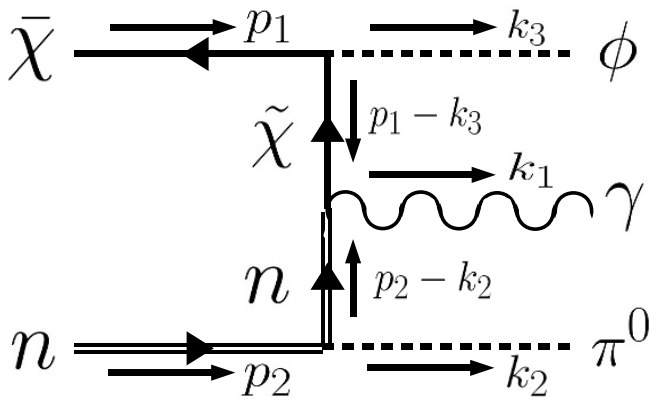

$a$.

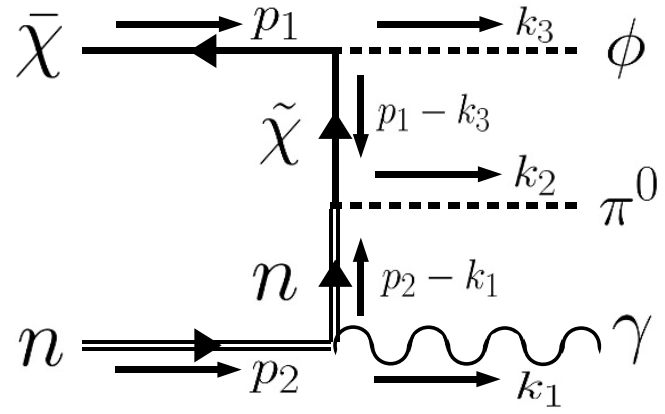

$b$.

Figure 2. Feynman diagrams for $\bar{\chi}+n \rightarrow \phi+\gamma+\pi^{0}$ in Model II.

which is the same as eq. (3.1) with $m_{\chi}$ replaced by $m_{\tilde{\chi}}$. Then $\chi$ couples to $\phi$ and $\tilde{\chi}$ via the new interaction,

$$
\mathcal{L} \subset \lambda_{\phi} \overline{\tilde{\chi}} \chi \phi+\text { h.c. }
$$

So, in addition to $n \rightarrow \gamma \tilde{\chi}$, a new neutron dark decay channel $n \rightarrow \phi \chi$ is allowed. Hence, the sum of the decay widths, $\Delta \Gamma_{n \rightarrow \gamma \tilde{\chi}}+\Delta \Gamma_{n \rightarrow \phi \chi} \simeq 7.1 \times 10^{-30} \mathrm{GeV}$, to reconcile the tension between beam and bottle experiments.

For $m_{\chi}>m_{\phi}$, the annihilation channel $\bar{\chi} \chi \rightarrow \phi \phi$ via $t$-channel $\tilde{\chi}$ exchange can provide the correct DM relic density if $\lambda_{\phi} \simeq 0.04$. The three masses $m_{\chi}, m_{\phi}$, and $m_{\tilde{\chi}}$ should satisfy the relations,

$$
\begin{aligned}
937.992 \mathrm{MeV} & <m_{\chi}+m_{\phi}<939.565 \mathrm{MeV}, \\
937.992 \mathrm{MeV} & <m_{\tilde{\chi}}, \\
\left|m_{\chi}-m_{\phi}\right| & <m_{p}+m_{e}=938.783081 \mathrm{MeV},
\end{aligned}
$$

to prevent ${ }^{9} \mathrm{Be}$ decays to ${ }^{8} \mathrm{Be}+\chi+\phi$ and ${ }^{8} \mathrm{Be}+\tilde{\chi}$, and to prohibit $\chi \rightarrow p+e^{-}+\bar{\nu}_{e}$, respectively $[8,9]$. We choose three benchmark points with $\lambda_{\phi} \simeq 0.04$ :

$$
\begin{array}{ll}
\text { P1 : } & \left(m_{\chi}, m_{\phi}, m_{\tilde{\chi}}\right)=(937.992,0,937.992) \\
\text { P2: } & \left(m_{\chi}, m_{\phi}, m_{\tilde{\chi}}\right)=\left(937.992,0,2 m_{n}\right) \\
\text { P3 : } & \left(m_{\chi}, m_{\phi}, m_{\tilde{\chi}}\right)=(939.174,0.391,940.000),
\end{array}
$$

where $m_{\chi} m_{\phi}$ and $m_{\tilde{\chi}}$ are in $\mathrm{MeV}$. All three points explain the neutron lifetime anomaly with the corresponding values of $\theta$ listed in table 1 . $\mathbf{P 1}$ and $\mathbf{P 2}$, respectively, are the points from refs. [7, 8], with $n \rightarrow \tilde{\chi} \gamma$ kinematically allowed for $\mathbf{P} 1$ but not for P2. Since $\tilde{\chi}$ plays the role of a propagator in the DM-neutron annihilation process, the signal event distributions are different for $\mathbf{P} \mathbf{1}$ and $\mathbf{P 2}$. For P3, the DM-neutron annihilation cross section is maximized, as we will see in the next section.

Feynman diagrams for the DM-neutron annihilation process,

$$
\bar{\chi}+n \rightarrow \phi+\gamma+\pi^{0},
$$




\begin{tabular}{|c|c|c|c|c|c|}
\hline & \multicolumn{2}{|c|}{ Model I } & $\mathbf{P 1}$ & P2 & P3 \\
\hline$m_{\chi}[\mathrm{MeV}]$ & 937.992 & 938.783 & 937.992 & 937.992 & 939.174 \\
\hline$m_{\phi}[\mathrm{MeV}]$ & - & - & 0 & 0 & 0.391 \\
\hline$m_{\tilde{\chi}}[\mathrm{MeV}]$ & - & - & 937.992 & $2 m_{n}$ & 940.000 \\
\hline$\lambda_{\phi}$ & - & -- & 0.04 & 0.04 & 0.04 \\
\hline$|\theta|$ & $6.14 \times 10^{-11}$ & $1.75 \times 10^{-10}$ & $4.21 \times 10^{-12}$ & $4.21 \times 10^{-12}$ & $4.03 \times 10^{-11}$ \\
\hline$\Gamma_{n \rightarrow \chi \gamma}($ or $\tilde{\chi} \gamma)[\mathrm{GeV}]$ & $7.1 \times 10^{-30}$ & $7.1 \times 10^{-30}$ & $3.3 \times 10^{-32}$ & 0 & 0 \\
\hline \multirow[t]{2}{*}{$\Gamma_{n \rightarrow \chi \phi}[\mathrm{GeV}]$} & - & - & $7.07 \times 10^{-30}$ & $7.10 \times 10^{-30}$ & $7.10 \times 10^{-30}$ \\
\hline & \multicolumn{2}{|c|}{$\bar{\chi} n \rightarrow \gamma \pi^{0}(y=2)$} & \multicolumn{3}{|c|}{$\bar{\chi} n \rightarrow \phi \gamma \pi^{0}(y=2)$} \\
\hline$\frac{v}{c} \sigma\left[\mathrm{cm}^{2}\right]$ & $6.83 \times 10^{-52}$ & $5.53 \times 10^{-51}$ & $5.02 \times 10^{-57}$ & $1.34 \times 10^{-57}$ & $3.02 \times 10^{-55}$ \\
\hline Super-K events & 6.72 & 54.4 & $4.9 \times 10^{-5}$ & $1.3 \times 10^{-5}$ & $3.0 \times 10^{-3}$ \\
\hline Hyper-K events & 163 & 1322 & $1.2 \times 10^{-3}$ & $3.2 \times 10^{-4}$ & $7.2 \times 10^{-2}$ \\
\hline \multirow[t]{2}{*}{ DUNE events } & 11.0 & 89.4 & $8.1 \times 10^{-5}$ & $2.2 \times 10^{-5}$ & $4.9 \times 10^{-3}$ \\
\hline & \multicolumn{2}{|c|}{$\bar{\chi} n \rightarrow 3 \pi^{0} \& 5 \pi^{0}$} & \multicolumn{3}{|c|}{$\bar{\chi} n \rightarrow \phi 3 \pi^{0}(y=0.542) \& \bar{\chi} n \rightarrow \phi 5 \pi^{0}(y=0.337)$} \\
\hline$\frac{v}{c} \sigma\left[\mathrm{cm}^{2}\right]$ & $9.71 \times 10^{-47}$ & $7.90 \times 10^{-46}$ & $2.51 \times 10^{-51}$ & $5.42 \times 10^{-54}$ & $7.04 \times 10^{-50}$ \\
\hline Super-K events & $9.59 \times 10^{5}$ & $7.78 \times 10^{6}$ & 24.7 & $5.4 \times 10^{-2}$ & 693 \\
\hline Hyper-K events & $2.32 \times 10^{7}$ & $1.88 \times 10^{8}$ & 601 & 1.30 & 16824 \\
\hline DUNE events & $1.57 \times 10^{6}$ & $1.28 \times 10^{7}$ & 40.7 & $8.8 \times 10^{-2}$ & 1137 \\
\hline
\end{tabular}

Table 1. Input parameters and observables for two benchmark points for Model I and points P1, P2 and P3 for Model II. Experimental cuts have not be applied.

are shown in figure 2. The event distributions will be different from Model I, due to the additional dark sector particle $\phi$ in the final state. Since $\phi$ can escape the detector, the reconstructed invariant mass $M_{\text {tot }}$ and total momentum $P_{\text {tot }}$ from $\gamma$ and $\pi^{0}$ have different distributions from Model I.

\section{Dark matter-nucleon annihilation cross section}

We now calculate the DM-neutron annihilation cross section for several possible signals in underground experiments. First, we determine the value of the mixing angle $\theta$ by requiring the neutron dark decay widths to be $\Delta \Gamma_{n \rightarrow \chi \gamma} \simeq 7.1 \times 10^{-30} \mathrm{GeV}$ and $\Delta \Gamma_{n \rightarrow \tilde{\chi} \gamma}+\Delta \Gamma_{n \rightarrow \chi \phi} \simeq$ $7.1 \times 10^{-30} \mathrm{GeV}$ for Models I and II, respectively. For Model II, we also fix $\lambda_{\phi}=0.04$, to obtain the correct DM relic density.

For the benchmark points in table 1, the typical values of the mixing angles are respectively, $\theta \simeq 10^{-10}$ and $10^{-11}$, in Model I and Model II. In general, $\theta$ in Model II is one order magnitude smaller than that in Model I, because

$$
\frac{\Delta \Gamma_{n \rightarrow \chi \gamma}}{\Delta \Gamma_{n \rightarrow \chi \phi}}=\frac{2 g_{n}^{2} e^{2}}{\left|\lambda_{\phi}^{2}\right|} \frac{\left(1-x_{1}^{2}\right)^{3}}{\sqrt{f\left(x_{1}, x_{2}\right)}}\left(\frac{m_{n}-m_{\tilde{\chi}}}{m_{n}-m_{\chi}}\right)^{2} \simeq \mathcal{O}\left(10^{-2}\right),
$$

where $f\left(x_{1}, x_{2}\right) \equiv\left[\left(1-x_{1}\right)^{2}-x_{2}^{2}\right]\left[\left(1+x_{1}\right)^{2}-x_{2}^{2}\right]^{3}$ with $x_{1} \equiv m_{\chi} / m_{n}$ and $x_{2} \equiv m_{\phi} / m_{n}$. 
The diagrams in figure 1 for $\bar{\chi} n \rightarrow \gamma \pi^{0}$ in Model I yield the spin averaged amplitude squared,

$$
\begin{aligned}
\frac{1}{4} \sum|M|^{2}= & \left|F_{\bar{\chi} \gamma n}\left(Q^{2}\right)\right|^{2}\left|F_{\bar{\chi} \pi n}\left(Q^{2}\right)\right|^{2} \\
& \times\left\{\frac{64 \pi g_{n \pi}^{2}}{\Lambda_{\chi n}^{2}} \frac{\left(m_{\chi}^{2}-t\right)\left[\left(s-m_{\pi}^{2}\right)\left(m_{\pi}^{2}+m_{n}^{2}-t\right)-m_{\pi}^{2}\left(m_{n}^{2}-u\right)\right]}{\left(t-m_{n}^{2}\right)^{2}}\right\},
\end{aligned}
$$

in terms of the Mandelstam variables $s \equiv\left(p_{1}+p_{2}\right)^{2}=\left(k_{1}+k_{2}\right)^{2}, t \equiv\left(p_{1}-k_{1}\right)^{2}=\left(p_{2}-k_{2}\right)^{2}$, and $u \equiv\left(p_{1}-k_{2}\right)^{2}=\left(p_{2}-k_{1}\right)^{2}$. In the static limit, which means the relative velocity of two initial particles approaches zero, the $\bar{\chi}$ and $n$ 4-momenta can be respectively approximated by $\left(m_{\chi}, \overrightarrow{0}\right)$ and $\left(m_{n}, \overrightarrow{0}\right)$, so that

$$
\begin{aligned}
\frac{1}{4} \sum|M|^{2}= & \left|F_{\bar{\chi} \gamma n}\left(Q^{2}\right)\right|^{2}\left|F_{\bar{\chi} \pi n}\left(Q^{2}\right)\right|^{2} \\
& \times\left\{\frac{64 \pi g_{n \pi}^{2}}{\Lambda_{\chi n}^{2}} m_{n} m_{\chi}\left(m_{n}+m_{\chi}\right)^{2}\left(\frac{\left(m_{n}+m_{\chi}\right)^{2}-m_{\pi}^{2}}{m_{n}\left(m_{n}+m_{\chi}\right)^{2}-m_{\chi} m_{\pi}^{2}}\right)\right\} .
\end{aligned}
$$

The cross section features a $1 / v$ behavior in the nonrelativistic limit (applicable for an average DM velocity $v \simeq 10^{-3} c$ ). We present values of $\frac{v}{c} \sigma$ in table 1 , which are independent of the DM velocity. This process produces $\gamma$ and $\pi^{0}$ at about a $\mathrm{GeV}$, which makes SuperKamiokande well suited to detect this signal.

We analytically estimate the form factor suppression for $\bar{\chi} n \rightarrow \gamma \pi^{0}$ as follows. While the virtual momentum flows in the two diagrams in figure 1 may be different, in the static limit they are the same: $-Q^{2}=\left(\frac{P}{2}-k_{1}\right)^{2}$. Therefore, the cross section is suppressed by the common factor,

$$
\left(\frac{1-m_{n}^{2} / \Lambda_{n}^{2}}{1+Q^{2} / \Lambda_{n}^{2}}\right)^{4 y}
$$

The maximum suppression $(y=2)$ for a typical value of $Q^{2}$ is $\mathcal{O}\left(10^{-5}\right)$. Note that the form factor suppression for $\bar{\chi} n \rightarrow \phi \gamma \pi^{0}$ in Model II depends on the kinematics and a full numerical integration is required. The values of $\frac{v}{c} \sigma$ including form factor suppression with $y=2$ are provided in table 1 .

Because Model II has multiple free parameters, $\left(m_{\chi}, m_{\phi}, m_{\tilde{\chi}}\right)$, the $\bar{\chi} n \rightarrow \phi \gamma \pi^{0}$ cross section is enhanced in some regions of parameter space. The maximum value of $\frac{v}{c} \sigma \simeq$ $\mathcal{O}\left(10^{-55}\right) \mathrm{cm}^{2}$ occurs at the corner of the parameter space, when three conditions are satisfied: i) $m_{\chi}+m_{\phi} \rightarrow 939.565 \mathrm{MeV}$, ii) $\left|m_{\chi}-m_{\phi}\right| \rightarrow m_{p}+m_{e}$, and iii) $m_{\tilde{\chi}} \rightarrow 937.992 \mathrm{MeV}$. P3 is such a benchmark point with $\sigma\left(\bar{\chi} n \rightarrow \phi \gamma \pi^{0}\right)=3.02 \times 10^{-55} \mathrm{~cm}^{2}$. The distribution of maximum and minimum cross section values in the $\left(m_{\chi}, m_{\phi}, m_{\tilde{\chi}}\right)$ parameter space are shown in figure 3. The benchmark points $\mathbf{P 1}, \mathbf{P 2}$, and $\mathbf{P 3}$ are marked with stars.

We now compute the DM-neutron annihilation cross section to multi- $\pi^{0}$. For Model I, the process is $\bar{\chi} n \rightarrow$ multi- $\pi^{0}$, and for Model II, $\phi$ is produced in association with multi- $\pi^{0}$, $\bar{\chi} n \rightarrow \phi+$ multi- $\pi^{0}$. Since the multi-pion channel is the dominant mode for antinucleusnucleus annihilation, we expect the same for DM-neutron annihilation.

Since a perturbation calculation is not valid for a large $\bar{n} \pi n$ coupling, $g_{n \pi} / \sqrt{4 \pi} \simeq \mathcal{O}(1)$, we use the experimentally measured value of the $\bar{n} p$ annihilation cross section in the low 

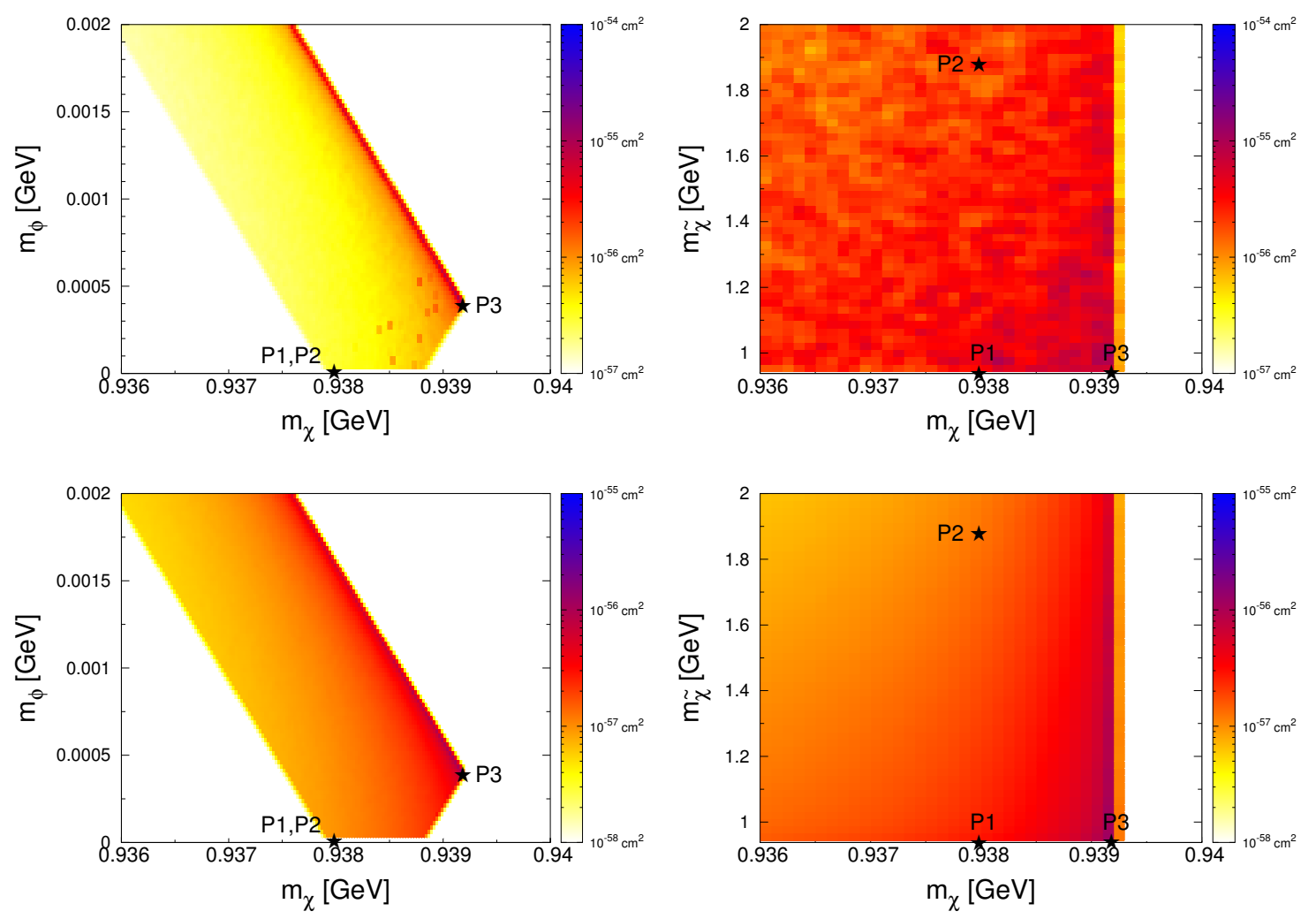

Figure 3. The maximum (upper panels), and minimum (lower panels) values of $\frac{v}{c} \sigma\left(\bar{\chi} n \rightarrow \gamma \pi^{0} \phi\right)$ projected on the $\left(m_{\chi}, m_{\phi}\right)$ plane (left panels), and the $\left(m_{\chi}, m_{\tilde{\chi}}\right)$ plane (right panels). The stars mark the three benchmark points P1, P2, and P3. We set $y=2$.

$\bar{n}$ velocity limit [19-22], obtained by extrapolating the cross section in ref. [19] to zero momentum:

$$
v \sigma(\bar{n} p \rightarrow \text { multi-pions })_{\exp }=44 \pm 3.5 \mathrm{mb},
$$

which is s-wave dominant and independent of the $\bar{n}$ velocity. We assume that $\sigma(\bar{n} n) \simeq$ $\sigma(\bar{n} p)$. Then for Model I, the DM-neutron annihilation cross section is given by

$$
v \sigma(\bar{\chi} n \rightarrow \text { multi-pions })=\theta^{2} v \sigma(\bar{n} p \rightarrow \text { multi-pions })_{\exp } .
$$

Numerical values for the sum of the cross section to the $3 \pi^{0}$ and $5 \pi^{0}$ channels, which have a total branching ratio of 0.585 [18], are provided in table 1.

For $\bar{\chi} n \rightarrow \phi+$ multi- $\pi^{0}$ in Model II, there is no experimental dataset that can be used directly. Our strategy is to perturbatively calculate $\bar{n} n \rightarrow$ pions cross sections and then require these to match Super-K's simulated cross sections in table I of ref. [18] by tuning the exponent $y$ of the form factor.

We find $y=0.542$ and $y=0.337$ for $\bar{n} n \rightarrow 3 \pi^{0}$ and $\bar{n} n \rightarrow 5 \pi^{0}$, respectively; see the appendix for a description of our procedure. Using these values of $y$ in eq. (2.2) and the mixing angle $\theta$, we calculate the cross sections for $\bar{\chi} n \rightarrow \phi 3 \pi^{0}$ and $\bar{\chi} n \rightarrow \phi 5 \pi^{0}$ as outlined in the appendix. Values for the sum of the cross section to these two channels are given in table 1. 


\section{Signal events at Super-K, Hyper-K and DUNE}

Armed with the $\bar{\chi} n$ annihilation cross section for different channels, the DM-nucleus annhilation cross section can be determined from $\sigma(\bar{\chi} A)=A^{2 / 3} \sigma_{0}[24]$, where $A$ is the atomic mass of the nucleus of atomic number $Z$, and $\sigma_{0}=\alpha \sigma(\bar{\chi} p)+(1-\alpha) \sigma(\bar{\chi} n)$, with $\alpha \equiv Z / A$. In the following, we make the assumption that $\sigma(\bar{\chi} p)=0$.

With water as the target for Super-K and Hyper-K, and $m_{\chi}=938.783 \mathrm{MeV}$, the interaction rate per second per gram of water is

$$
n_{\chi} v_{\mathrm{DM}}\left[\left(N_{A} \cdot 1 / 18\right) \cdot \sigma(\bar{\chi} O)+\left(N_{A} \cdot 2 / 18\right) \cdot \sigma(\bar{\chi} H)\right],
$$

where $v_{\mathrm{DM}}=10^{-3} c$ is the thermal average DM velocity, the DM number density is $n_{\chi}=$ $\rho_{\chi} / m_{\chi}$ per $\mathrm{cm}^{3}$ in terms of the local DM density $\rho_{\chi}=0.3 \mathrm{GeV} / \mathrm{cm}^{3}, N_{A}=6.022 \times 10^{23}$ is the Avogadro number, and $N_{A} / 18$ is the total number of $\mathrm{H}_{2} \mathrm{O}$ molecules per gram of water. For the liquid Argon target at DUNE, the interaction rate per second per gram of target is

$$
n_{\chi} v_{\mathrm{DM}}\left[\left(N_{A} / 40\right) \cdot \sigma(\bar{\chi} A r)\right] / \rho_{\mathrm{Ar}}
$$

where $\rho_{\mathrm{Ar}}=1.3954 \mathrm{~g} / \mathrm{cm}^{3}$ is the density of liquid Argon. In the nonrelativistic limit, the interaction rate is independent of the $v_{\mathrm{DM}}$, and therefore independent of the velocitydistribution of the DM in the galactic halo.

The signal events are obtained by multiplying the above interaction rates with the total exposure. For Super-K [17], the current total exposure is 306.3 kiloton-years. For Hyper-K [25], we use a fiducial mass of 372 kiloton with 20 years of data-taking. For DUNE [26], we take a 40 kiloton fiducial mass with 20 years of data-taking. The events numbers for the different signal channels in the three experiments are displayed in table 1.

The kinematic cuts applied in Super-K's searches for proton decay and $n-\bar{n}$ oscillations are summarized in table 2 . We adopt the same cuts (cut-1, cut-2, cut-3) and definitions of total visible momentum, $P_{\text {tot }} \equiv\left|\sum_{i}^{\text {all-rings }} \overrightarrow{p_{i}}\right|$, where $\overrightarrow{p_{i}}$ is the reconstructed momentum vector of the $i^{\text {th }}$ ring, the invariant mass, $M_{\text {tot }} \equiv \sqrt{E_{\text {tot }}^{2}-P_{\text {tot }}^{2}}$, and the total visible energy, $E_{\text {tot }} \equiv \sum_{i}^{\text {all-rings }} \sqrt{p_{i}^{2}+m_{i}^{2}}$, where $m_{i}$ is the mass of the $i^{\text {th }}$ ring assuming that showering and nonshowering rings are from $\gamma$ and $\pi^{ \pm}$, respectively [18]. For our case, $m_{i}=0$. Kinematic cut-1 was applied for the $n-\bar{n}$ oscillation search for which the observed number of events $N_{\text {obs }}=24$ is consistent with the number of background events $N_{\text {bkgd }}=24.1$. Correspondingly, the $3 \sigma$ range of the allowed number of signal events is $N_{\text {Super-K }}^{3 \sigma} \subset[0,22.5]$ [27]; the allowed number of signal events for cut-2 and cut-3 are as in table 2. To evaluate the expected number of signal events at Hyper-K and DUNE, we assume that the observed event rate is compatible with the expected background rate, and scale Super-K's exposure. The $3 \sigma$ ranges are provided in table 2.

To calculate the number of events that satisfy the kinematic cuts, we perform a Monte Carlo simulation by assuming 10\% momentum uncertainty for each ring in Super$\mathrm{K}$ [28]. ${ }^{1}$ We take the momentum resolution at Hyper-K and DUNE to be $10 \%$. The

\footnotetext{
${ }^{1}$ The momentum resolution is estimated to be $0.6+2.6 \sqrt{\mathrm{P}(\mathrm{GeV} / \mathrm{c})} \%$ for Super-K [28]. Since for our signal processes, each ring has about a few hundred $\mathrm{MeV}$ in energy, we simply adopt a $10 \%$ momentum resolution.
} 


\begin{tabular}{|c|c|ccc|c|c|}
\hline & Kinematic cuts (in MeV) & $N_{\text {obs }}$ & $N_{\text {bkgd }}$ & $N_{\text {Super-K }}^{3 \sigma}$ & $N_{\text {Hyper-K }}^{3 \sigma}$ & $N_{\text {DUNE }}^{3 \sigma}$ \\
\hline cut-1 & $P_{\text {tot }} \subset[0,450] M_{\text {tot }} \subset[750,1800][18]$ & 24 & 24.1 & {$[0,22.5]$} & {$[0,75]$} & {$[0,27]$} \\
\hline cut-2 & $P_{\text {tot }} \subset[0,100], M_{\text {tot }} \subset[800,1050][17]$ & 0 & 0.07 & {$[0,7]$} & {$[0,5.5]$} & {$[0,4]$} \\
\hline cut-3 & $P_{\text {tot }} \subset[100,250], M_{\text {tot }} \subset[800,1050][17]$ & 0 & 0.54 & {$[0,6.5]$} & {$[0,7]$} & {$[0,5.8]$} \\
\hline
\end{tabular}

Table 2. Three kinematic regions from $n-\bar{n}$ oscillations [18] and proton decay [17] searches at Super-K. $N_{\text {Super-K }}^{3 \sigma}$ is the allowed number of signal events within $3 \sigma$. The $3 \sigma$ expectation for the number of signal events at Hyper-K and DUNE is obtained under the assumption that the observed number of events is compatible with the number of background events.
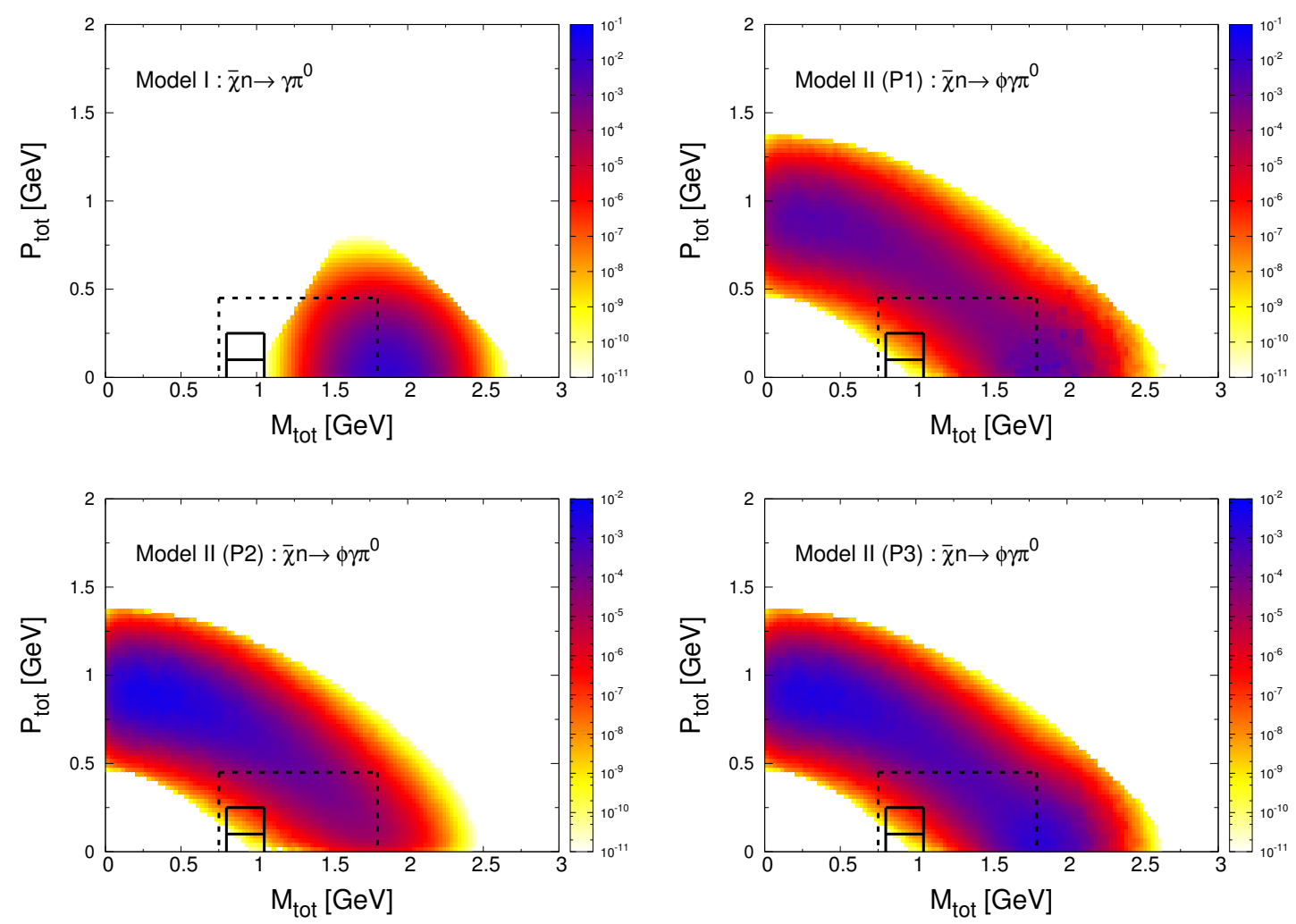

Figure 4. The normalized signal event distributions for $\bar{\chi} n \rightarrow \gamma \pi^{0}$ and $\bar{\chi} n \rightarrow \phi \gamma \pi^{0}$ in the $\left(M_{\text {tot }}, P_{\text {tot }}\right)$ plane for Model I (with $m_{\chi}=937.992 \mathrm{MeV}$ ) and the three benchmark points of Model II. The dashed rectangle corresponds to kinematic cut-1, while two solid lower and upper rectangles correspond to cut-2 and cut-3, respectively.

event distributions projected on to the $\left(M_{\text {tot }}, P_{\text {tot }}\right)$ plane for $\bar{\chi} n \rightarrow \gamma \pi^{0}\left(\phi \gamma \pi^{0}\right)$ and $\bar{\chi} n \rightarrow \operatorname{multi}-\pi^{0}\left(\phi+\right.$ multi- $\left.\pi^{0}\right)$ are shown in figures 4 and 5 , respectively. Kinematic cut- 1 is the region within the dashed rectangle, and cut-2 (cut-3) is within the solid lower (upper) rectangle. In figure 4 , we show the $\bar{\chi} n \rightarrow \gamma \pi^{0}$ event distribution for the $m_{\chi}=937.992 \mathrm{MeV}$ case of Model I, but the distribution is not visibly changed for the $m_{\chi}=938.783 \mathrm{MeV}$ case. The $\bar{\chi} n \rightarrow \phi \gamma \pi^{0}$ event distributions are shown for points P1, P2, and P3 of Model II; less then $20 \%$ of the total events fall in the cut-1 region. Because the kinematic distributions of our multi- $\pi^{0}$ signals are similar to that of $n-\bar{n}$ oscillations, in figure 5 , a majority of 

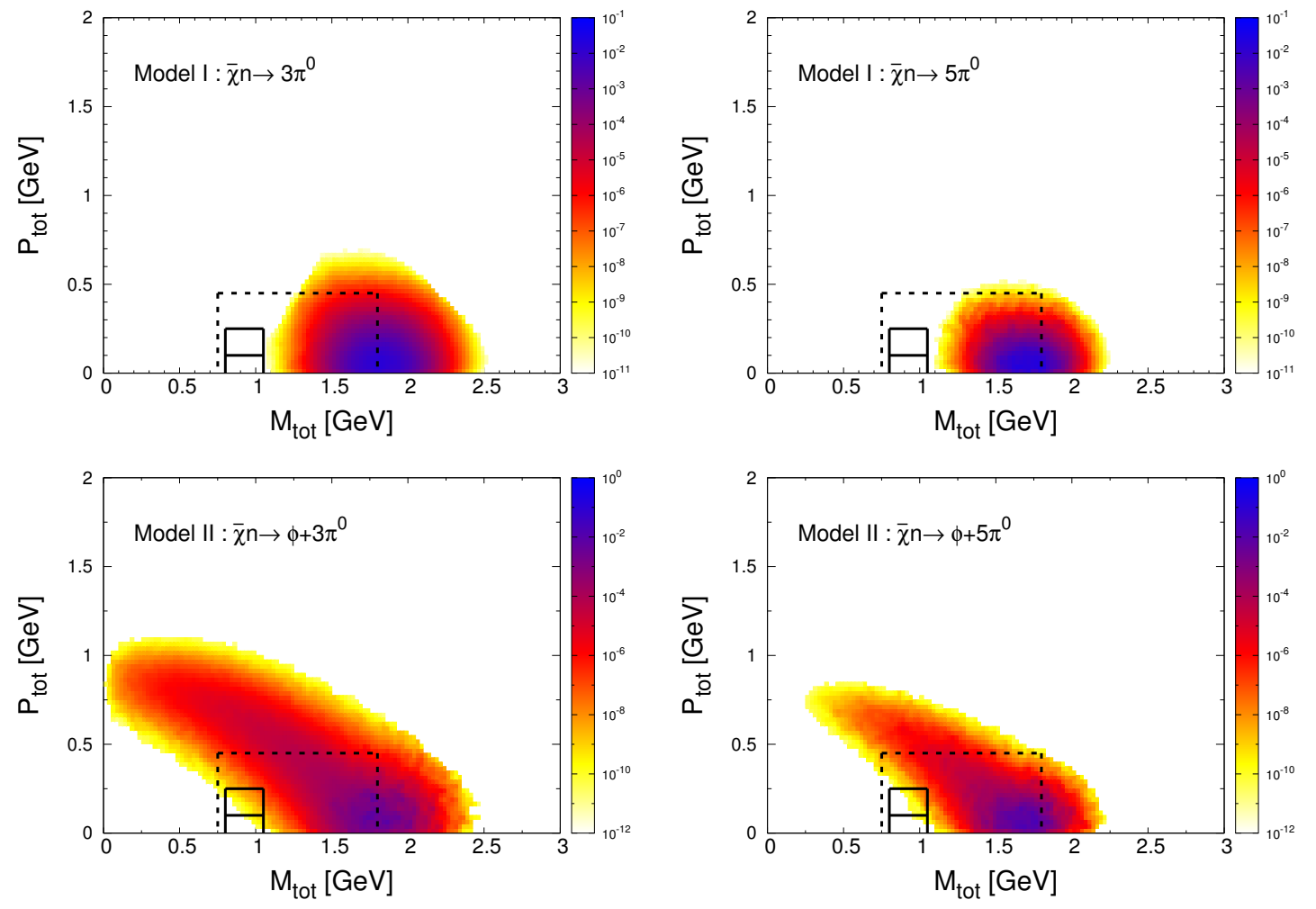

Figure 5. The normalized signal event distributions for $\bar{\chi} n \rightarrow 3 \pi^{0}\left(5 \pi^{0}\right)$ for Model I (with $m_{\chi}=$ 937.992 MeV) and $\bar{\chi} n \rightarrow \phi 3 \pi^{0}\left(\phi 5 \pi^{0}\right)$ for point P1 of Model II.

\begin{tabular}{|c|ccc|ccc|}
\hline & \multicolumn{3}{|c|}{ Model I: $m_{\chi}=937.992 \mathrm{MeV}$} & \multicolumn{3}{c|}{ Model II: P1 } \\
\hline & $\bar{\chi} n \rightarrow \gamma \pi^{0}$ & $\bar{\chi} n \rightarrow 3 \pi^{0}$ & $\bar{\chi} n \rightarrow 5 \pi^{0}$ & $\bar{\chi} n \rightarrow \phi \gamma \pi^{0}$ & $\bar{\chi} n \rightarrow \phi 3 \pi^{0}$ & $\bar{\chi} n \rightarrow \phi 5 \pi^{0}$ \\
\hline cut-1 & $31.2 \%$ & $41.0 \%$ & $79.7 \%$ & $15.4 \%$ & $78.3 \%$ & $71.8 \%$ \\
cut-2 & $2.9 \times 10^{-9} \%$ & $1.1 \times 10^{-9} \%$ & $5.7 \times 10^{-9} \%$ & $2.4 \times 10^{-6} \%$ & $2.4 \times 10^{-7} \%$ & $1.5 \times 10^{-7} \%$ \\
cut-3 & $2.7 \times 10^{-10} \%$ & $5.7 \times 10^{-10} \%$ & $1.0 \times 10^{-10} \%$ & $3.8 \times 10^{-4} \%$ & $2.3 \times 10^{-5} \%$ & $1.5 \times 10^{-5} \%$ \\
\hline & \multicolumn{3}{|c|}{ Model II: P2 } & \multicolumn{3}{c|}{ Model II: P3 } \\
\hline & $\bar{\chi} n \rightarrow \phi \gamma \pi^{0}$ & $\bar{\chi} n \rightarrow \phi 3 \pi^{0}$ & $\bar{\chi} n \rightarrow \phi 5 \pi^{0}$ & $\bar{\chi} n \rightarrow \phi \gamma \pi^{0}$ & $\bar{\chi} n \rightarrow \phi 3 \pi^{0}$ & $\bar{\chi} n \rightarrow \phi 5 \pi^{0}$ \\
\hline cut-1 & $1.76 \%$ & $57.6 \%$ & $93.5 \%$ & $14.6 \%$ & $57.5 \%$ & $87.3 \%$ \\
cut-2 & $1.3 \times 10^{-6} \%$ & $7.8 \times 10^{-6} \%$ & $4.6 \times 10^{-6} \%$ & $3.2 \times 10^{-6} \%$ & $1.0 \times 10^{-6} \%$ & $3.6 \times 10^{-7} \%$ \\
cut-3 & $2.8 \times 10^{-4} \%$ & $1.1 \times 10^{-3} \%$ & $1.1 \times 10^{-3} \%$ & $5.0 \times 10^{-4} \%$ & $1.0 \times 10^{-4} \%$ & $5.8 \times 10^{-5} \%$ \\
\hline
\end{tabular}

Table 3. Percentage of events that pass the kinematic cuts.

the events fall inside the cut-1 region, and only a tiny fraction of events are inside the cut-2 and cut-3 regions. Therefore, these signals do not contaminate the proton decay search. In table 3, we tabulate the percentage of events for each channel that pass the three kinematic cuts. 


\section{Results and summary}

Under the assumption that $\bar{\chi}$ is stable on the scale of the age of universe and is the dominant component of dark matter, Model I is comfortably ruled out by the current $n-\bar{n}$ oscillation search at Super-K because it predicts $\mathcal{O}\left(10^{6}\right) \bar{\chi} n \rightarrow$ multi- $\pi^{0}$ events in the cut-1 region, while Super-K has observed 24 events with an expected background of 24.1 events. Note that theoretical uncertainties do not affect this exclusion because the calculation of the $\bar{\chi} n \rightarrow$ multi- $\pi^{0}$ cross section is driven by experimental data, and so is not impacted by the hadron form factor uncertainty.

It is difficult to completely explore the parameter space of Model II because of its many degrees of freedom, and hence difficult to rule it out. We therefore focused on specific benchmark points. The expected numbers of $\phi 3 \pi^{0}+\phi 5 \pi^{0}$ signal events for $\mathbf{P 1}$, P2, and P3 at Super-K after applying cut-1 are 18.1, 0.040, and 545, respectively, where we used $y=0.542$ and $y=0.337$ for $\bar{\chi} n \rightarrow \phi 3 \pi^{0}$ and $\bar{\chi} n \rightarrow \phi 5 \pi^{0}$, respectively. It is clear that $\mathbf{P} \mathbf{3}$ is excluded by Super-K at more than $3 \sigma$ for the above values of $y$. P1 does not contribute a significant event excess at Super-K. P2 is three orders of magnitude beyond the reach of Super-K because of the heavier $\tilde{\chi}$.

If cut-1 is extended to $M_{\text {tot }}=2 \mathrm{GeV}$, the signal events increase to $24.4,0.041$, and 680 for P1, P2, and P3, respectively, and more than $95 \%$ of the signal events fall inside the extended kinematic region for $\mathbf{P} \mathbf{1}$ and $\mathbf{P} 3$.

We show the sensitivities of Super-K and the future experiments Hyper-K and DUNE in terms of $y$ in table 4, where we applied kinematic cut-1. The table gives the minimum value of $y$ that ensures that the number of signal events lies within the $3 \sigma$ range in table 2 . Negative values of $y$ mean that although there is no form factor suppression, the experiment cannot probe the parameter point. Clearly, DUNE will have better sensitivity than Super-K, and Hyper-K will have the best sensitivity as evidenced by the higher minimum values of $y$.

\section{Acknowledgments}

W.-Y.K. and P.-Y.T. thank the National Center of Theoretical Sciences, Taiwan, for its hospitality. D.M. is supported in part by the U.S. DOE under Grant No. de-sc0010504. P.-Y.T. is supported by World Premier International Research Center Initiative (WPI), MEXT, Japan.

\section{A Cross section calculations}

We describe the procedure to obtain the $\bar{\chi} n \rightarrow \phi 3 \pi^{0}$ and $\bar{\chi} n \rightarrow \phi 5 \pi$ cross sections including the form factor suppressions.

From a theoretical perspective, it is difficult to calculate the antineutron-neutron cross section and branching ratios because non-perturbative pion interactions are involved. Instead, we extract the annihilation cross sections and branching ratios from relevant experimental data, and then use them to determine the unknown exponent $y$ in the form factor 


\begin{tabular}{|c|c|c|c|}
\hline \multicolumn{4}{|c|}{ Super-K } \\
\hline & $\mathbf{P 1}$ & P2 & P3 \\
\hline $\bar{\chi} n \rightarrow \phi \gamma \pi^{0}$ & -0.807 & -3.48 & -0.236 \\
\hline $\bar{\chi} n \rightarrow \phi 3 \pi^{0}$ & 0.229 & -0.721 & 0.883 \\
\hline $\bar{\chi} n \rightarrow \phi 5 \pi^{0}$ & 0.260 & -0.502 & 0.735 \\
\hline \multicolumn{4}{|c|}{ Hyper-K } \\
\hline & P1 & P2 & P3 \\
\hline $\bar{\chi} n \rightarrow \phi \gamma \pi^{0}$ & -0.434 & -2.88 & 0.172 \\
\hline $\bar{\chi} n \rightarrow \phi 3 \pi^{0}$ & 0.658 & -0.371 & 1.297 \\
\hline $\bar{\chi} n \rightarrow \phi 5 \pi^{0}$ & 0.535 & -0.261 & 1.003 \\
\hline \multicolumn{4}{|c|}{ DUNE } \\
\hline & P1 & P2 & P3 \\
\hline $\bar{\chi} n \rightarrow \phi \gamma \pi^{0}$ & -0.751 & -3.38 & -0.173 \\
\hline $\bar{\chi} n \rightarrow \phi 3 \pi^{0}$ & 0.296 & -0.665 & 0.948 \\
\hline $\bar{\chi} n \rightarrow \phi 5 \pi^{0}$ & 0.304 & -0.464 & 0.777 \\
\hline
\end{tabular}

Table 4. The minimum value of $y$ for Model II that produces a signal event number within the $3 \sigma$ range in table 2; the maximum value (which gives 0 events) is $y=\infty$. Here kinematic cut-1 is applied.

of eq. (2.2). Higher-order corrections, pion self interactions, and form factor uncertainties are embedded in this form factor. The values of $y$ can be different for different final states because our "effective form factor" includes the aforementioned effects. We emphasize that our form factor is not one that underlies the fundamental process that gives different final states.

According to ref. [19], $v \sigma(\bar{n} n \rightarrow$ pions $)=v \sigma(\bar{n} p \rightarrow \text { pions })_{\exp }=44$ mb at zero momentum, where we have assumed that the $\bar{n} n$ annihilation cross section is the same as the $\bar{n} p$ cross section. We checked this result by fitting the data in ref. [19]; see figure 6 . Using the branching ratios, $\operatorname{Br}\left(\bar{n} n \rightarrow \pi^{+} \pi^{-} \pi^{0}\right)=0.065$ and $\operatorname{Br}\left(\bar{n} n \rightarrow\left(\pi^{+} \pi^{-} 3 \pi^{0}\right)+\left(2 \pi^{+} 2 \pi^{-} \pi^{0}\right)\right)=$ $0.28+0.24=0.52$ [18], which were obtained from $\bar{p} p$ and $\bar{p} d$ bubble chamber data, we find the numerical values of $\sigma\left(\bar{n} n \rightarrow 3 \pi^{0}\right)=0.065 \cdot \sigma(\bar{n} n \rightarrow$ pions $)$ and $\sigma\left(\bar{n} n \rightarrow 5 \pi^{0}\right)=$ $0.52 \cdot \sigma(\bar{n} n \rightarrow$ pions $)$. Next, we determine $y$ by comparing our Monte Carlo calculations with the values for $\sigma\left(\bar{n} n \rightarrow 3 \pi^{0}\right)$ and $\sigma\left(\bar{n} n \rightarrow 5 \pi^{0}\right)$. To calculate the leading order cross sections, we implement the neutron-pion interaction in the CalcHEP package [23], generate the amplitude squared, and insert the form factor of eq. (2.2) by hand. To evaluate $\sigma\left(\bar{n} n \rightarrow 3 \pi^{0}\right)(y)$ and $\sigma\left(\bar{n} n \rightarrow 5 \pi^{0}\right)(y)$, where we have made the $y$-dependence explicit, we perform the three-body and five-body phase space integrations, respectively. Matching the cross sections, $\sigma\left(\bar{n} n \rightarrow 3 \pi^{0}\right)(y)=\sigma\left(\bar{n} n \rightarrow 3 \pi^{0}\right)$ and $\sigma\left(\bar{n} n \rightarrow 5 \pi^{0}\right)(y)=\sigma\left(\bar{n} n \rightarrow 5 \pi^{0}\right)$, yields $y=0.542$ and $y=0.337$, respectively.

Using the above values of $y$, we calculate $\sigma\left(\bar{\chi} n \rightarrow \phi 3 \pi^{0}\right)$ and $\sigma\left(\bar{\chi} n \rightarrow \phi 5 \pi^{0}\right)$ for Model II, by replacing the initial particle $\bar{n}$ by $\bar{\chi}$, multiplying by the mixing parameter $\theta$, and including the dark sector particle $\phi$ in the final state. We implement the $\chi-n-\phi$ interaction in CalcHEP, output the amplitude squared, convolve with the form factor, and perform the four-body and six-body phase space integrations. 


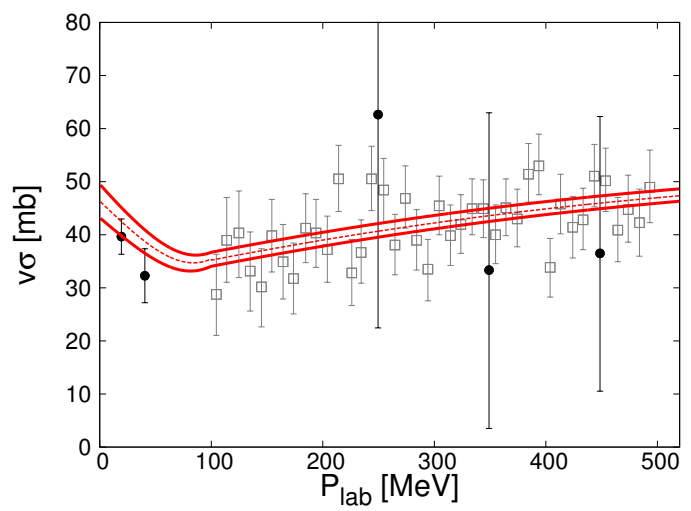

Figure 6. A fit to the data in figure 14 of ref. [19]. The red dashed curve is the best fit with $\chi^{2} /$ dof $=0.74$, and the red solid curves show the $1 \sigma$ allowed range. The annihilation cross section at zero momentum from our fit is consistent with that of ref. [19]: $v \sigma=44 \pm 3.5 \mathrm{mb}$.

Open Access. This article is distributed under the terms of the Creative Commons Attribution License (CC-BY 4.0), which permits any use, distribution and reproduction in any medium, provided the original author(s) and source are credited.

\section{References}

[1] A. Pichlmaier, V. Varlamov, K. Schreckenbach and P. Geltenbort, Neutron lifetime measurement with the UCN trap-in-trap MAMBO II, Phys. Lett. B 693 (2010) 221 [INSPIRE].

[2] A. Steyerl et al., Quasielastic scattering in the interaction of ultracold neutrons with a liquid wall and application in a reanalysis of the Mambo I neutron-lifetime experiment, Phys. Rev. C 85 (2012) 065503 [INSPIRE].

[3] S. Arzumanov et al., A measurement of the neutron lifetime using the method of storage of ultracold neutrons and detection of inelastically up-scattered neutrons, Phys. Lett. B 745 (2015) 79 [INSPIRE].

[4] J. Byrne and P.G. Dawber, A revised value for the neutron lifetime measured using a penning trap, Europhys. Lett. 33 (1996) 187 [INSPIRE].

[5] A.T. Yue et al., Improved determination of the neutron lifetime, Phys. Rev. Lett. 111 (2013) 222501.

[6] Particle Data Group, Review of particle physics, Chin. Phys. C 40 (2016) 100001.

[7] B. Fornal and B. Grinstein, Dark matter interpretation of the neutron decay anomaly, Phys. Rev. Lett. 120 (2018) 191801 [arXiv:1801.01124] [INSPIRE].

[8] B. Fornal and B. Grinstein, Neutron lifetime discrepancy as a sign of a dark sector?, arXiv: 1810.00862 [INSPIRE].

[9] M. Pfützner and K. Riisager, Examining the possibility to observe neutron dark decay in nuclei, Phys. Rev. C 97 (2018) 042501 [arXiv:1803.01334] [INSPIRE].

[10] M. Jin and Y. Gao, Nucleon-light dark matter annihilation through baryon number violation, Phys. Rev. D 98 (2018) 075026 [arXiv: 1808.10644] [INSPIRE]. 
[11] D. McKeen, A.E. Nelson, S. Reddy and D. Zhou, Neutron stars exclude light dark baryons, Phys. Rev. Lett. 121 (2018) 061802 [arXiv: 1802 . 08244] [INSPIRE].

[12] G. Baym, D.H. Beck, P. Geltenbort and J. Shelton, Testing dark decays of baryons in neutron stars, Phys. Rev. Lett. 121 (2018) 061801 [arXiv:1802.08282] [INSPIRE].

[13] T.F. Motta, P.A.M. Guichon and A.W. Thomas, Implications of neutron star properties for the existence of light dark matter, J. Phys. G 45 (2018) 05LT01 [arXiv:1802.08427] [INSPIRE].

[14] B. Grinstein, C. Kouvaris and N.G. Nielsen, Neutron star stability in light of the neutron decay anomaly, arXiv:1811.06546 [INSPIRE].

[15] J.J. de Swart, M.C.M. Rentmeester and R.G.E. Timmermans, The status of the pion-nucleon coupling constant, PiN Newslett. 13 (1997) 96 [nucl-th/9802084] [INSPIRE].

[16] Y. Aoki, T. Izubuchi, E. Shintani and A. Soni, Improved lattice computation of proton decay matrix elements, Phys. Rev. D 96 (2017) 014506 [arXiv:1705.01338] [InSPIRE].

[17] Super-Kamiokande collaboration, Search for proton decay via $p \rightarrow e^{+} \pi^{0}$ and $p \rightarrow \mu^{+} \pi^{0}$ in 0.31 megaton-years exposure of the Super-Kamiokande water Cherenkov detector, Phys. Rev. D 95 (2017) 012004 [arXiv: 1610.03597] [INSPIRE].

[18] Super-Kamiokande collaboration, The search for $n-\bar{n}$ oscillation in Super-Kamiokande I, Phys. Rev. D 91 (2015) 072006 [arXiv:1109.4227] [INSPIRE].

[19] G.S. Mutchler et al., Measurement of the imaginary part of the $I=1 \bar{N} N$ S-wave scattering length, Phys. Rev. D 38 (1988) 742 [inSPIRE].

[20] OBELIX collaboration, Total and annihilation $\bar{n} p$ cross-sections from $50 \mathrm{MeV} / \mathrm{c}$ to $400 \mathrm{MeV} / c$, Nucl. Phys. A 655 (1999) 224 [INSPIRE].

[21] OBELIX collaboration, $\bar{n} p$ annihilation in flight in two mesons in the momentum range between $50 \mathrm{MeV} / \mathrm{c}$ and $400 \mathrm{MeV} / \mathrm{c}$ with OBELIX, Nucl. Phys. Proc. Suppl. 56 (1997) 227 [INSPIRE].

[22] BROOKHAVEN-HOUSTON-PENNSYLVANIA STATE-RICE collaboration, Measurement of anti-neutron proton total and annihilation cross-sections from $100 \mathrm{MeV} / \mathrm{c}$ to $500 \mathrm{MeV} / \mathrm{c}$, Phys. Rev. D 36 (1987) 659 [InSPIRE].

[23] A. Belyaev, N.D. Christensen and A. Pukhov, CalcHEP 3.4 for collider physics within and beyond the Standard Model, Comput. Phys. Commun. 184 (2013) 1729 [arXiv:1207.6082] [INSPIRE].

[24] M. Astrua et al., Antineutron nucleus annihilation cross-sections below $400 \mathrm{MeV} / \mathrm{c}, \mathrm{Nucl}$. Phys. A 697 (2002) 209 [INSPIRE].

[25] Hyper-Kamiokande collaboration, Hyper-Kamiokande design report, arXiv: 1805.04163 [INSPIRE].

[26] DUNE collaboration, Long-Baseline Neutrino Facility (LBNF) and Deep Underground Neutrino Experiment (DUNE), arXiv:1512.06148 [INSPIRE].

[27] G.J. Feldman and R.D. Cousins, A unified approach to the classical statistical analysis of small signals, Phys. Rev. D 57 (1998) 3873 [physics/9711021] [INSPIRE].

[28] Super-Kamiokande collaboration, A measurement of atmospheric neutrino oscillation parameters by Super-Kamiokande I, Phys. Rev. D 71 (2005) 112005 [hep-ex/0501064] [INSPIRE]. 